\title{
Submillimeter-Scale Analysis and Machine Learning For the Generation of Alginate Hydrogel Beads
}

\author{
Peiyuan $\mathrm{He}^{1}$, Liguo Zhang ${ }^{2, \text { a, }}{ }^{*}$ \\ ${ }^{1}$ School of Life Science, Zhengzhou University, Zhengzhou, China \\ 2Institute of Medical and Pharmaceutical Science, Zhengzhou University, Zhengzhou, China \\ axinxin201403@sina.com
}

Keywords: microbeads at submillimter-scale; manipulated its fabrication.

\begin{abstract}
The fabrication of microbeads at submillimter-scale has been focused on as the scientific research topic since they have been widely applied in various kinds of industries, such as pharmacology, cosmetics as well as food industry. The development of applied mathematics, material technology and information technology accelerated the submillimeter-scale analysis on the generation behaviors for the hydrogel microbeads and afterwards the manipulated its fabrication. In this work, the experiments were designed to manipulate hydrogel microbeads. The statistical analysis was carried out, the nonlinear modeling on the variation of microbeads was undertaken and its improved version was created. Submillimeter analysis-based machine learning technology was finally introduced to test and verified the neural network modelling stisfactorily.
\end{abstract}

\section{Introduction}

Alginate is a natural polysaccharide derived from marine plant and its basic structure consists of linear unbranched polymers containing $\beta$-1,4-linked D-mannuronic(M) and $\alpha$-1,4-linked L-guluronic $(G)$ residues. It can form thermally stable and biocompatible hydrogel in the presence of calcium cation[1]. As the hydrogel can be easily produced into particulate form by using simple method, it has been used in many encapsulation applications such as in the field of biomedical, bioprocess, pharmaceutical, and food[2]. Examples of the encapsulated compound are such as cells[3], microbial cells[4], drugs, oils [5] and flavors[6].

The design of alginate particle is highly dependent on the applications. The parameters such as size, size distribution, shape, porosity, mechanical properties are considerable. The characters of alginate particles are mainly influenced by calcium cation concentration[7] and alginate concentration[8] during the gelation process. In some industrial process, the production of alginate particle is always needed to be in critical consistency, which is not only the dosage requirement, but also the quality assurance and aesthetical art.

The way to produce spherical alginate particle is typically by using the extrusion technique[9]. The alginate solution is extruded through a needle, and is allowed to drip into the calcium solution. Alginate gel particle is formed in the calcium solution. The form of the gel particle has a relation with the surface tension of calcium solution, alginate solution concentration, and the tip size and etc. if these parameters are not proper, the gel particle formed in the calcium solution will have a tail. Hence, It is necessary to develop a model to descript the relationship between these parameters and the produced spherical gel particles. In the second part, the experiments were orthogonally designed based on two essential parameters for the manipulation of microbeads. The statistical analysis on the key experimental factors was carried out to express the generalization of different trial levels. In the third part, the nonlinear modeling, proved effective, on the variation of microbeads was undertaken and its improved version was created. Submillimeter analysis-based machine learning technology was finally introduced to test and verified the neural network modelling stisfactorily. 


\section{Experiments and Analytical Modeling}

\subsection{Experiment Design}

Through our precedent research and publications, two essential factors, the concentration of alginate solution and the needle diameter, play key role in the manipulation of alginate hygrogel particle sizes at certain flow rate[10]. Thus the investigation was designed to clarify the role of the two parameters by observing the phenomena under their possible combinative conditions. The concentration $\mathrm{C}$ was designed to include five levels, with a specific value each, as shown in Table 1; the needle diameter D consisted of three levels, each level with a particular value, shown in Table 2. Experiment conditions were distributed uniformly in the research space, shown in Figure 1. The hydrogel particles size $\mathrm{S}$ variation, presented as its diameter, can be readily compared through this experimental configuration.

Table 1. Three $\mathrm{C}$ levels for corresponding values

\begin{tabular}{cccccc}
\hline Level & $\mathbf{1}$ & $\mathbf{2}$ & $\mathbf{3}$ & $\mathbf{4}$ & $\mathbf{5}$ \\
\hline Value & 0.03 & 0.045 & 0.06 & 0.075 & 0.09 \\
\hline
\end{tabular}

Table 2. Three D levels for corresponding values

\begin{tabular}{ccccc}
\hline Level & $\mathbf{1}$ & $\mathbf{2}$ & $\mathbf{3}$ \\
\hline Value & 0.6 & 0.7 & 0.8 \\
\hline $\mathbf{2} 2$ Fabrication
\end{tabular}

\subsection{Fabrication of Microparticles}

Micro-beads are made of a alginate (A0682,Sigma Aldrich, USA) solution at five different concentration, varying from $3 \%$ to $9 \%$ with the $1.5 \%$ interval increases. The alginate solution is extruded through three different gauge needles, $0.6 \mathrm{~mm}, 0.7 \mathrm{~mm}, 0.8 \mathrm{~mm}$ (Fisher Scientific), which can define the bead size by a peristaltic pump (Ismatec ISM834C,Switzerland) at a flow rate of $5 \mathrm{ul} / \mathrm{min}$. Hydrogel particles of alginate solution are formed at the tip of the needle. When the hydrogel particle gravity is greater than the adhesion force of the alginate solution, the hydrogel particles fall into a 50 $\mathrm{ml}$ calcium chloride solution at a concentration of $1.7 \%$. They are left in the calcium solution for 20 minutes so as to polymerize. The distance between the needle tip and the cation solution surface is 10 $\mathrm{cm}$ to obtain spherical hydrogel particles. The beads are stored in the $1.7 \%$ calcium chloride solution to stabilize the alginate gel.

\subsection{Descriptive Analysis}

Descriptive analysis for the hydrogel particle variation was undertaken to see the effects of $\mathrm{C}$ and $\mathrm{D}$, respectively. The results presented the relation between the hydrogel particle size $\mathrm{S}$ as function of $\mathrm{C}$ and D under submillimeter-scale experimental conditions. S seemed to be on the direct proportion to $\mathrm{C}$, while almost in the same tendancy for D. This experimental observations can be logically explained: for the increase of $\mathrm{C}$, the central viscous force became stronger, which took effects to give more time for hydrogel particle formation, permitting to enlarge hydrogel particle size; Similarly, the augmentation of D led to stabilize larger hydrogel particles, allowing for the increase of hydrogel particle sizes. We find the variation of hydrogel particle size for $\mathrm{C}$ is 1.64 times as large as that of $\mathrm{D}$. The $\mathrm{C}$ seemed to weigh more for hydroge particle size variation in this investigation.

\subsection{Correlation Analysis}

The correlation analysis was performed between $\mathrm{C}$ and D. C and D were found to be independent to each other, with.This is a logic result for the two experimental conditions were separately manipulated between $\mathrm{C}$ and $\mathrm{D}$. This finding proposed a possibility to include these two flow rates into the mathematical model to express their behaviors for hydrogel particle generation. 


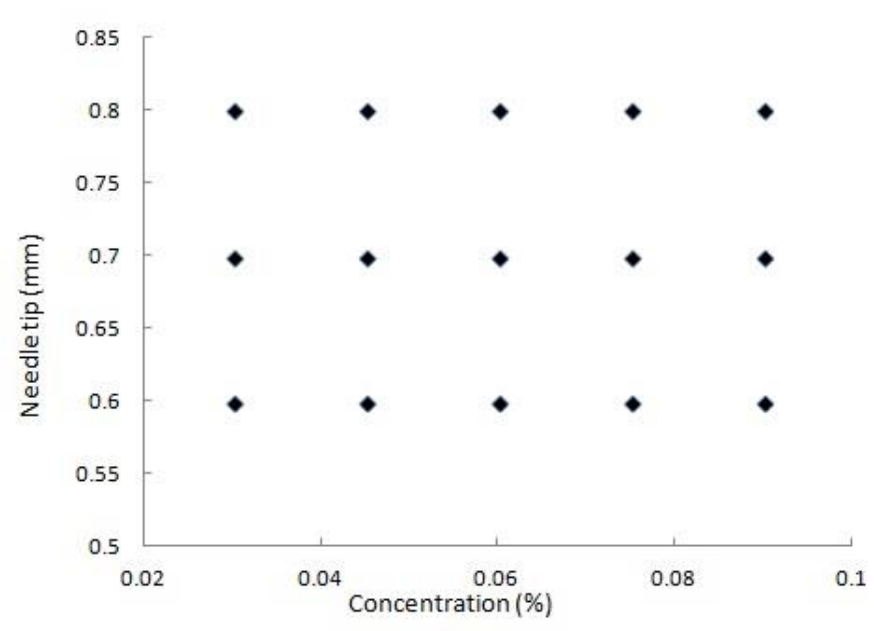

\subsection{Nonlinear Modeling}

Figure 1. Experimental conditions

As shown in Equation 1, the nonlinear model showed that as increase of $\mathrm{C}$ hydrogel particle sizes could increase. The same phenomena were observed through different experiments. This result could be explained through mechanical viewpoint: In this submillimeter scale, surface tension and viscous force take main effects. Surface tension between hydrogel solution and air tended to interrupt droplet enlargement and facilitated the hydrogel particle formation, while the fluidic viscous force took more effects on keeping this process stable. The adjustment of $\mathrm{C}$ and $\mathrm{D}$ led to the formation of different hydrogel particles. Besides, the greater $\mathrm{C}$ supported stronger viscous effects, permitting to enlarge the droplet at the tip more; while with the augmentation of needle diameter $\mathrm{D}$, the stable droplet formation condition can be achieved, resulting in larger hydrogel particles. The same phenomena were observed through different manipulations.

$$
\mathrm{S}=2439 \times \frac{\mathrm{C}}{\mathrm{D}}+1180, \mathrm{R}^{2}>0.48
$$

However, this nonlinear model seemed to some extent to be controdictory to the experimental observation. The unsatisfactory explanation consisted in that, with the increases of $\mathrm{D}$, the particle sizes were expected to decrease in the model; but this expectation were opposite to the experimental observation. This contradiction between the theoretical model and the experimental result can be attributed to the imcompleteness of model establishment. This defective model can somewhat explain the hydrogel droplet generation, but obviously needed to be well improved. The coefficient R2 value presented that this model had something short of effectiveness. The hydrogel particle generation was regarde as a complex nonlinear process involving the interactions between physico-chemical property and mechanical forces. Then more parameter should be involved to optimize the nonlinear modeling. For the defectiveness lied in the needle diameter $\mathrm{D}$, the item $\mathrm{D}$ was tested in the nonlinear model to see the effectiveness of needle diameter.

\subsection{Optimization of Nonlinear Model}

The modeling for hydrogel particle generation was optimized with the inclusion of needle diameter D, as presented in Equation. 2. The improved nonlinear model could explain well the hydrogel particle size variation.

$$
\mathrm{S}=3178 \times \frac{\mathrm{C}}{\mathrm{D}}+941 \times \mathrm{D}+457 \mathrm{R}^{2}>0.89
$$

This improved nonlinear model showed that as the increase of $\mathrm{C}$ or $\mathrm{D}$, mathematically, the hydrogel particle sizes increased for the experimental ranges; besides, with the increase of flow rates item $\frac{C}{D}$, hydrogel particle sizes varied towards the same direction. For C, hydrogel particle sizes varying as the same results as former nonlinear model; For D, however, hydrogel particle sizes varied differently comparing with the defective nonlinear model. This difference consisted in the inclusion 
of linear item D, showing that the involvement of D could take effects on each item at the model. The addition of linear item improved the model quality but also expressed the descriptive complexity for the hydrogel particle variation. The conclusion deduced from this model coincided well with the experimental observations.

\subsection{Machine Learning and Modeling Verification}

Machine learning is the effective tool for the description and prediction to nonliear behaviors, not only in macroacale phenomena, but in the submillimeter as well [11,12]. In this study, the Neural Network Modeling (NNM) for hydrogel particle size variation was performed. The C and D were contained in the input layer, while the responses in this non-linear model represented the hydrogel particle size. The theoretical analysis was performed to compare the values extracted from experiments with the theoretical ones calculated through neural networks modeling. The validated results were presented in the Figure 2. These predicted values fell in the vicinity of the experimental curve, demonstrating the effectiveness of this modeling based on the machine learning technology.

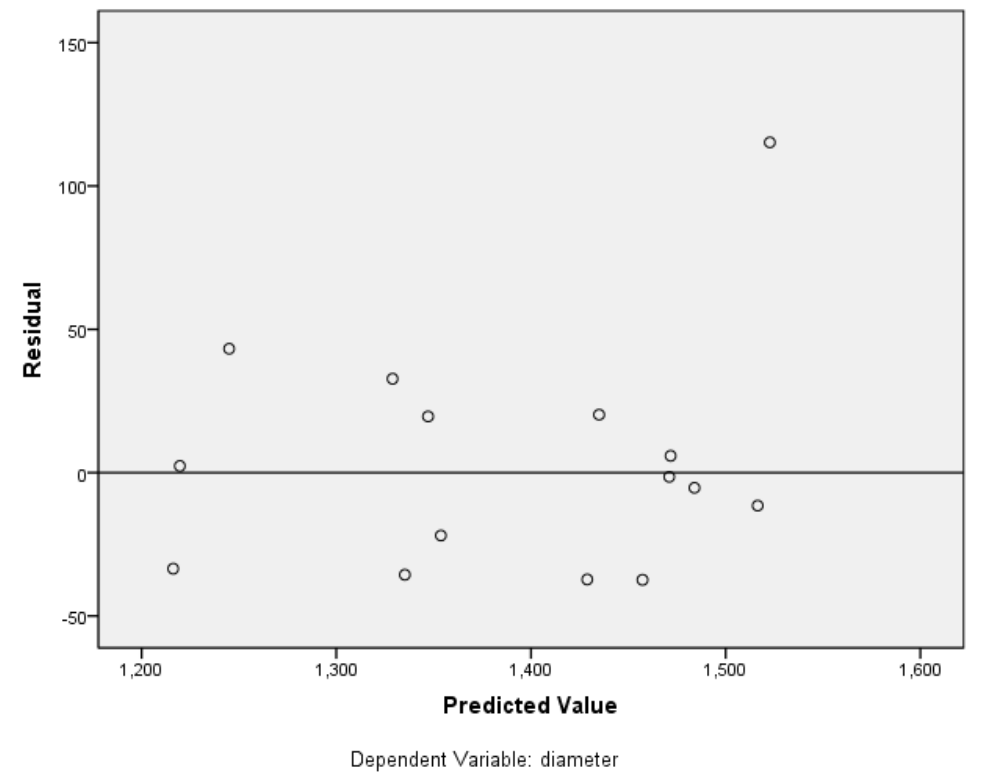

Figure 2. Verification of ANN models

\section{Conclusion}

For this investigation, the descriptive analysis and numerical modeling were performed. The inside mechanism in biomicrofludic hydrogel particle generation were clarified through different numerical modeling. Specifically, the role for two independent factors $\mathrm{C}$ and $\mathrm{D}$ was examined. The $\mathrm{C}$ was found to weigh more than $\mathrm{D}$ in hydrogel particle variation. The information mining from experimental samples facilitated the establishment of the numerical models. The simple nonlinear model could present the defective description for the hydrogel droplet generation. The non-linear optimizations ameliorated the descriptive satisfaction of numerical model. Machine learning technology, artificial neural network, was introduced to simulate the hydrogel particle variations. After the verification procedure, it was demonstrated that machine learning brought satisfactory accuracy to the hydrogel particle variation.

\section{Acknowledgments}

This work was supported by the Key Program for Science and Technology Development of education department of Henan province (No. 17A180036, 17A180037), and by the natural science foundation of Henan province (No.172102310004), by National Natural Science Foundation of China (No.31600676). 


\section{References}

[1]. Vicini S, Castellano M, Mauri M, Marsano E, Gelling process for sodium alginate: New technical approach by using calcium rich micro-spheres. Carbohydrate Polymers 134 (2015):767-774.

[2]. Agüero L, Zaldivar-Silva D, Peña L, Dias ML Alginate microparticles as oral colon drug delivery device: A review. Carbohydrate Polymers.

[3]. Hashemi M, Kalalinia F, Application of encapsulation technology in stem cell therapy. Life Sciences 143(2015):139-146.

[4]. Vemmer M, Patel AV, Review of encapsulation methods suitable for microbial biological control agents. Biological Control 67 (2013):380-389.

[5]. Rodríguez J, Martín MJ, Ruiz MA, Clares B, Current encapsulation strategies for bioactive oils: From alimentary to pharmaceutical perspectives. Food Research International 83 (2016):41-59.

[6]. Ghodke S, Bhanvase B, Sonawane S, Mishra S, Joshi K, 16 - Nanoencapsulation and nanocontainer based delivery systems for drugs, flavors, and aromas A2 - Grumezescu, Alexandru Mihai. In: Encapsulations. Academic Press, 2016, pp 673-715.

[7]. Çelik E, Bayram C, Akçapınar R, Türk M, Denkbaş EB, The effect of calcium chloride concentration on alginate/Fmoc-diphenylalanine hydrogel networks. Materials Science and Engineering: C 66 (2016):221-229.

[8]. Mohanty S, Wu Y, Chakraborty N, Mohanty P, Ghosh G, Impact of alginate concentration on the viability, cryostorage, and angiogenic activity of encapsulated fibroblasts. Materials Science and Engineering: C 65 (2016):269-277.

[9]. Davarc1 F, Turan D, Ozcelik B, Poncelet D, The influence of solution viscosities and surface tension on calcium-alginate microbead formation using dripping technique. Food Hydrocolloids 62 (2017):119-127

[10]. He P, Zhang L Multi-dimensional Analysis, Information Mining and Numerical Modeling for Small Samples of Biomedical Microfluidic Droplets. Biomedical Science \& Engineering 3 (1) (2015):1-3

[11]. Zhou L, Pan S, Wang J, Vasilakos AV, Machine learning on big data: Opportunities and challenges. Neurocomputing 237 (2017):350-361.

[12]. Aldhaheri M, Wei M, Bai B, Alsaba M (2017) Development of machine learning methodology for polymer gels screening for injection wells. Journal of Petroleum Science and Engineering 151:77-93. doi:http://dx.doi.org/10.1016/j.petrol.2016.12.038 